\section{Therapie der Spastizität mit Botulinumtoxin Typ A}

Jörg Wissel1, Markus Naumann²

\author{
1 Neurologische Rehabilitationsklinik, Kliniken Beelitz GmbH \\ 2 Neurologische Klinik, Augsburg
}

psychoneuro 2005; 31 (6): 300-306

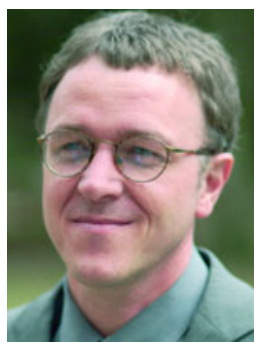

Jörg Wissel
Lokale intramuskuläre Injektionen mit Botulinumtoxin Typ A werden heute als Behandlung erster Wahl in der Therapie von fokalen dystonen und spastischen Syndromen eingesetzt. Durch die eintretende Tonusreduktion können behindernde Bewegungsstörung und assoziierte Schmerzen über drei bis sechs Monate deutlich reduziert werden. Pflege und Körperhygiene der Betroffenen werden erleichtert, die Lebensqualität nimmt zu. Neben den zugelassenen Indikationen, wie Spasmus hemifacialis, Blepharospasmus, Hand-oder Armspastik bei erwachsenen Schlaganfallpatienten, wird aufgrund der Datenlage auch der Einsatz bei weiteren fokalen Behinderungen durch Spastizität empfohlen. Der Einsatz von Botulinumtoxin bei Spastik an den unteren und oberen Extremitäten wird beschrieben.

S pastizität ist durch eine inadäquate und überschießende Muskelaktivität gekennzeichnet. Definitionsgemäß wird darunter eine geschwindigkeitsabhängige Steigerung des Muskeltonus verstanden (13). Die pathologische Tonussteigerung kann in Ruhe fehlen und ist um so ausgeprägter, je rascher der Muskel passiv gedehnt wird. Ist die überschießende Muskelaktivität spontan und anhaltend, liegt eine sogenannte spastische Dystonie vor. Spastizität kann bei vielen neurologischen Erkrankungen auftreten, die mit einer Schädigung des kortikospinalen Traktes einschließlich der begleitenden extrapyramidalen Bahnsysteme verbunden sind, z.B. nach Schlaganfall, Schädel-Hirn-Traumen, entzündlichen Erkrankungen des Nervensystems, Multiple Sklerose, hypoxischen Hirnschädigungen und Rückenmarksläsionen. Unbehandelt führt schwere Spastizität zu funktionell relevanten Motilitätseinschränkungen, chronischen Schmerzen und verringerter Lebensqualität der Betroffenen (10).
Zur Therapie stehen neben Physiotherapie, die medikamentöse Therapie mit oralen Antispastika (z.B. Baclofen, Tizanidin oder Benzodiazepine), die intrathekale Therapie mit Baclofen oder die Injektion von Botulinumtoxin zur Verfügung.

Botulinumtoxin ist die lokale Behandlung der Wahl bei fokalen behindernden Dystonien und anderen Muskelspasmen (Torticollis spasmaticus und Blinzelkrämpfen und $\mathrm{He}-$ mispasmus facialis). Bei fokalen Dystonien, die auf eine oder wenige Körperregionen begrenzt sind, sind orale Medikamente nur unzureichend wirksam. Gute Erfolge werden dagegen mit Botulinumtoxin erreicht, das sich seit Ende der 80er Jahre als effektive und nebenwirkungsarme Therapieoption etabliert hat (17).

\section{Wirkmechanismus \\ Botulinumtoxin}

Das Zellgift Botulinumtoxin wird von dem Bacterium Clostridium botulinum produziert. Von den sieben bekannten Serotypen (Typ A bis G) werden dabei therapeutisch nur die
Toxine vom Typ A (Botox ${ }^{\circledR}$, Dysport ${ }^{\circledR}$, Xeomin $^{\circledR}$ ) und Typ B (NeuroBloc $\left.{ }^{\circledR}\right)$ genutzt. Durch die intramuskuläre Injektion werden hochselektiv cholinerge Synapsen blockiert und damit die Freisetzung von Acetylcholin an der motorischen Endplatte gehemmt, der Muskel erschlafft (8). Der Muskeltonus wird so in der Regel je nach Injektionsort und -menge zwischen zwei bis sechs Monaten dosisabhängig anhaltend reduziert.

Botulinumtoxin führt durch die gehemmte Acetylcholinausschüttung an der motorischen Endplatte zu einer reversiblen dosisabhängigen chemischen Denervierung. Nach der intramuskulären Injektion wird das Toxin in den präsynaptischen Anteil des terminalen Axons aufgenommen und bewirkt als Zink-abhängige Protease eine Blockierung der Ausschleusung der acetylcholinbeladenen Vesikel in den synaptischen Spalt. Es kommt so zu einer dosisabhängigen Lähmung der extrafusalen Muskulatur mit einer ebenso dosisabhängigen Reduktion der Spastizität. Eine spastische Fehlstellung aufgrund einer muskulären Überaktivität sowie begleitende Schmerzen können daher verringert werden. Die bei chronischer Spastizität auftretende Verkürzung der Muskellänge der betroffenen Muskulatur wird durch den Einsatz von Botulinumtoxin und passive oder aktive Dehnung der behandelten spastischen Muskeln verbessert. Durch die Blockade der muskelspindelregulierenden intrafusalen Mus- 
kulatur werden gleichzeitig die sogenannten IA-Muskelspindelafferenzen zu spinalen reflexmodulierenden Zentren reduziert, d.h. neben der Tonusminderung mit Parese der behandelten Muskulatur wird der afferente neuronale Einstrom aus der behandelten Region zu spinalen Reflex- und Regulationszentren moduliert. Möglicherweise kann die gestörte reziproke Aktivierung und zusätzlich die Antagonistenaktivität günstig beeinflusst werden.

\section{- Indikationen/Therapieziele}

Generell gilt, dass Botulinumtoxin bei Bewegungsstörungen am besten wirkt, bei denen es sich um einen exakt umschriebenen fokal begrenzten Bereich der spastischen Bewegungsstörung handelt. Durch die Injektion kann z.B. eine Tonusreduktion der spastischen Ellbogen-, Hand- und Fingerbeuger, der Flexoren des Sprunggelenks und der Zehen sowie im Kieferbereich erreicht werden. Zugelassen sind die bisher auf dem Markt verfügbaren Botulinumtoxine allerdings bisher nur für Spasmus hemifacialis (Botox ${ }^{\circledR}$, Dysport $^{\circledR}$ ), Blepharospasmus (Botox ${ }^{\circledR}$, Dysport $^{\circledR}$, Xeomin $^{\circledR}$ ), idiopathische rotatorische zervikale Dystonie (Torticollis spasmodicus: Botox ${ }^{\circledR}$, Dysport $^{\circledR}$, Xeomin $^{\circledR}$, Neurobloc $^{\circledR}$ ), fokale Spastizität mit dynamischer Spitzfußstellung infolge von Spastizität bei Patienten mit infantiler Zerebralparese, die zwei Jahre und älter sind (Botox $\left.{ }^{\circledR}\right)$, Hand- und Handgelenkspastik bei erwachsenen Schlaganfallpatienten (Botox ${ }^{\circledR}$ ) und Armspastik nach Schlaganfall (Dysport ${ }^{\circledR}$ ) sowie in der Indikation „starke, fortbestehende primäre Hyperhidrosis axillaris, die störende Auswirkungen auf die Aktivitäten des täglichen Lebens hat und mit einer topischen Behandlung nicht ausreichend kontrolliert werden kann“ (Botox ${ }^{\circledR}$.

Außerdem wird die Therapie mit Botulinumtoxin Typ A aufgrund der Studienlage und der vorliegenden Erfahrungen zurzeit von der Deutschen Gesellschaft für Neurorehabilitation (DGNR) und dem Arbeitskreis Botulinumtoxin der Deutschen Gesellschaft für Neurologie bei folgenden Indikationen empfohlen (1):
- bei Arm- oder Handspastik anderer Ursache als durch Schlaganfall bzw. bei Beinspastik auch aus anderen Ursachen als durch infantile Zerebralparese. Solche anderen Ursachen sind beispielsweise Schädel-Hirn-Traumen, Hirnentzündungen (z.B. Multiple Sklerose), Hirntumoren oder Schädigungen bzw. Erkrankungen des Gehirns und Rückenmarks bei spastischer Spitzfußund Inversionsstellung, die sich durch Schienen, orale Antispastika und physikalische Maßnahmen nicht beheben lässt

- oro-mandibulärer oder lingualer Dystonie (19)

- laryngealer Dystonie (Spasmodischer Dysphonie) (5)

- Extremitätendystonien an Bein/ Fuß und Arm/Hand insbesondere aufgabenspezifische Dystonien (z.B. Schreibkrampf, Musikerdystonie) (7)

- Rumpfdystonien (z.B. Kamptokormie) (16)

- neben axillärer Hyperhidrose auch bei Frey-Syndrom, palmarer Hyperhidrose und Hypersalivation sowie Hyperlakrimation.

Ausschlusskriterien für eine Botulinumtoxin-Behandlung sind Schwangerschaft und Stillperiode, schwere Gerinnungsstörungen, fixierte knöcherne Kontraktur und schwere neuromuskuläre Erkrankungen sowie bekannte lokale oder systemische allergische Reaktion auf Botulinumtoxin.

Der Behandlungserfolg ist abhängig von der Erfahrung des behandelnden Arztes mit Botulinumtoxin und Bewegungsstörungen, Injektionsmenge und Injektionsstelle sowie vom Injektionszeitpunkt. Botulinumtoxin Typ A sollte eingesetzt werden, bevor sich eine behindernde Abnahme der Muskellänge bei Spastizität mit Kontrakturen entwickelt. Idealerweise sollte die Behandlung in ein multimodales Konzept eingebunden sein, an dem Arzt, Patient, ggf. Angehörige, Physio- und Ergotherapeuten sowie Pflegepersonal beteiligt sind.

Das Behandlungsziel richtet sich nach der vorliegenden Symptomatik und den Erfordernissen des Patien- ten. Im Vordergrund stehen die Reduktion von begleitenden Schmerzen, die Erleichterung von Pflege und Körperhygiene sowie die Verbesserung einer motorischen Funktion. Außerdem kann das Ausmaß von bestehenden, nicht knöchernen Kontrakturen verringert und Kontrakturen vermieden werden (20).

Funktionelle Verbesserungen der oberen und unteren Extremitäten sind möglich, können jedoch häufig nicht erreicht werden. Mitunter kann aufgrund des klinischen Befunds nur schwierig zwischen fixierten und dynamischen Kontrakturen differenziert werden, dann kann z.B. eine Nervenblockade mit Lokalanästhetika oder auch eine probatorische Botulinumtoxin-A-Injektion sinnvoll sein.

\section{Spastizität der oberen Extremität}

Bei der spastischen Bewegungsstörung der oberen Extremität findet sich bei Erwachsenen mit supratentoriellem Hirninfarkt häufig das sogenannte spastische WernickeMann-Muster: Innenrotation und Adduktion des Oberarms, Beugung im Ellbogenbereich mit Pronation des Unterarms, Flexion des Handgelenks und der Langfinger sowie Flexion und Adduktion des Daumens. Schulter- und Ellenbogengelenk können bei schwerer Spastizität meist nur langsam oder in vielen Fällen überhaupt nicht mehr aktiv bewegt werden. Die Hand ist oft spastisch zusammengeballt oder kann nur noch zum Halten und Fixieren von Gegenständen eingesetzt werden. Diese Bewegungsstörungen werden von den Patienten als erheblich stigmatisierend erlebt. Der flektierte Ellbogen und das flektierte Handgelenk erschweren das Anund Auskleiden. Bei starker Fingerund Daumenflexion der geschlossenen Hand ist das Fingelnägelschneiden und die Reinigung zwischen den Fingern und der Handinnenflächen beeinträchtigt. In der Folge können Pilz- und bakterielle Infektionen entstehen. Darüber hinaus kann eine chronische Druckeinwirkung auf die Haut der Handinnenfläche zu Mazerationen führen. Die assoziierten Schmerzen verstärken die Spas- 


\section{Tab. 1 Spastizität der oberen Extremität mit Dosierungsempfehlungen}

\begin{tabular}{|lllll} 
Muskel & $\begin{array}{l}\text { Anzahl der } \\
\text { Injektions- } \\
\text { stellen }\end{array}$ & $\begin{array}{l}\text { Dosis Botox } \\
\text { (Units) }\end{array}$ & $\begin{array}{l}\text { Dosis Dysport } \\
\text { (Units) }\end{array}$ & $\begin{array}{l}\text { Dosis Xeomin }^{\circledR} \\
\text { (Units) }\end{array}$ \\
\hline M. deltoideus & 2 & $25-75$ & $125-350$ & $25-75$ \\
\hline M. pectoralis major & $3-4$ & $50-100$ & $250-400$ & $50-100$ \\
\hline M. teres major & $1-2$ & $25-50$ & $125-250$ & $25-50$ \\
\hline M. biceps brachii & $3-4$ & $50-100$ & $250-500$ & $50-100$ \\
\hline M. brachialis & 2 & $25-50$ & $125-250$ & $25-50$ \\
\hline M. brachioradialis & 2 & $25-50$ & $125-250$ & $25-50$ \\
\hline M. flex. carpi radialis & 2 & $25-50$ & $125-250$ & $25-50$ \\
\hline M. flex. carpi ulnaris & 2 & $25-50$ & $125-250$ & $25-50$ \\
\hline M. flex. dig. profundus & $2-3$ & $25-50$ & $125-250$ & $25-50$ \\
\hline M. flex. dig. superfi. & $2-3$ & $25-50$ & $125-250$ & $25-50$ \\
\hline M. flexor poll. Longus & 2 & $25-50$ & $125-250$ & $25-50$ \\
\hline M. adductor pollicis & 1 & $15-25$ & $100-125$ & $15-25$ \\
\hline Maximale Gesamtdosis & & 400 & 1000 & 300 \\
\hline Empfohlene Verdünnungen & $2-5 \mathrm{ml} /$ & $2-4 \mathrm{ml} /$ & $2-5 \mathrm{ml} /$ \\
& 100 Units & 500 Units & 100 Units \\
\hline
\end{tabular}

${ }^{*}$ Xeomin ${ }^{\circledR}$ nicht zugelassen in der Behandlung der Spastizität der oberen Extremität Die angegebenen Dosierungen sind Richtwerte, die sich auf persönliche Erfahrungen und Literaturangaben stützen
Form einer Tonusminderung und Mobilitätsverbesserung vor. Eine erneute Injektion ist dann häufig wirksamer als die erste. Bei einer repetitiven Behandlung z.B. der spastischen Flexorenhaltung der Finger und der Hand nach Schlaganfall, scheint die Wirksamkeit weiter zuzunehmen (12). Allgemein werden daher Injektionen in einem DreiMonats-Intervall empfohlen. Aus Kosten- und Verträglichkeitsgründen sollten bei der oberen Extremität dabei nicht mehr als 400 Units Botox $^{\circledR}$ und 1000 Units Dysport ${ }^{\circledR}$ eingesetzt werden. Pro Injektionsstelle wird empfohlen, nicht mehr als 50 Units Botox ${ }^{\circledR}$ und 250 Units Dysport ${ }^{\circledR}$ zu injizieren (21). Der Abstand zwischen zwei Injektionen sollte mindestens acht Wochen betragen, um sogenannte „Booster“Injektionen mit der erhöhten Gefahr der Bildung von neutralisierenden Antikörpern zu verhindern.

Eine Injektionskontrolle mittels Elektrostimulation oder Sonografie wird empfohlen, der Einsatz der EMG wird bei der Spastizität der oberen Extremitäten unterschiedlich bewertet (siehe Beitrag von Ceballos-Baumann in diesem Heft). Bei aktionsspezifischen Dystonien, z.B. Schreibkrampf, bei denen sich oftmals nach Injektionen einzelner Unterarmmuskeln eine verbesserte Funktionalität der Hand erreichen lässt, wird dagegen häufig ein EMG zur Injektionskontrolle herangezogen. Bei Injektionen in den M. biceps brachii und $\mathrm{M}$. brachioradialis ist dies dagegen oft nicht erforderlich. Bei gewünschter hoher Selektivität der Injektion und verminderter Kooperation bei sedierten Patienten wird eine durch Elektrostimulation kontrollierte Injektion von Botulinumtoxin empfohlen.

\section{Spastizität der unteren Extremität}

Bei der Spastizität der unteren Extremitäten wird Botulinumtoxin am häufigsten bei spastischem Spitzfuß (Pes Equinus) eingesetzt. Dabei ist vorwiegend der funktionelle Strecker des oberen Sprunggelenkes (Plantarflexoren) betroffen. Die Patienten gehen meist auf den Zehenspitzen mit primärem Vorfußkontakt 
in der initialen Standbeinphase ohne weiteres Abrollen des Fußes. Liegt dabei ein hoher dynamischer Anteil an der Spitzfußhaltung vor, der ein geringes Maß an strukturellen, bindegewebigen Veränderungen des Muskels wahrscheinlich macht, werden mit BTA gute Ergebnisse erzielt. Das Gangbild und die Gehgeschwindigkeit können sich unter der Therapie verbessern $(3,18)$, wobei auch physiotherapeutische Maßnahmen und Redressionen in die Therapie mit einbezogen werden sollten. Die Behandlung sollte dabei möglichst früh begonnen werden, da sekundäre ossäre Folgeschäden verringert werden können. Insbesondere bei Kindern unter sechs Jahren kann zudem die Notwendigkeit eines orthopädischen Eingriffs bis in ein späteres Lebensalter ( $>6$ Jahre) verschoben werden, in dem der Eingriff in der Regel ein geringeres Rezidivrisiko zeigt. In Deutschland ist bisher nur Botox $^{\circledR}$ für die Behandlung des Pes Equinus ab dem zweiten Lebensjahr zugelassen. Im Erwachsenenalter muss jedoch trotz erwiesener Wirksamkeit in plazebokontrollierten Studien in dieser Indikation der Einsatz in Deutschland noch im Sinne einer sogenannten „off label“ Behandlung durchgeführt werden (10).

Zielmuskeln bei Spastizitäten der unteren Extremität (Tab. 2) sind bei Spitzfuß der M. gastrocnemius medial und lateral auch der M. soleus, außerdem bei Inversion und Supination der M. tibialis posterior. Sind Extensoren beteiligt, kann auch eine Injektion in den M. tibialis anterior indiziert sein. Bei Kindern mit infantiler Zerebralparese und Spitzfuß sollte in jedem Fall der M. gastrocnemius mediale und laterale und gegebenenfalls $\mathrm{M}$. soleus und $\mathrm{M}$. tibialis posterior behandelt werden.

Bei spastischen Zehenfehlstellungen, z.B. der striatalen Großzehe, werden mit Injektionen in die betroffenen Muskeln (M. extensor hallucis longus, M. flexor digitorum longus und brevis) gute Erfolge erzielt.

Eine Injektionskontrolle ist bei Injektionen in den $\mathrm{M}$. gastrocnemius medialis und lateralis nicht unbedingt erforderlich. Bei Kindern mit Zerebralparese kann zur exak- ten Muskellokalisation allerdings eine sonografiekontrollierte Injektion sinnvoll sein. Dieses Vorgehen wird auch bei der Injektion in den M. soleus, M. tibialis posterior und M. flexor digitorum longus empfohlen.

Die Wirkdauer beträgt über drei Monate, repetitive Injektionen alle drei bis vier Monate führen oft zu einer weiteren kontinuierlichen messbaren Funktionsverbesserung.

\section{Adduktorenspastizität}

Eine Adduktorenspastik ist eine häufige Indikation für eine Botulinumtoxin-Behandlung bei spastischen Bewegungsstörungen im Kindes- und Erwachsenenalter. Dabei werden die Beine von den Adduktoren mit einer großen Kraftentfaltung in eine Scherenstellung gezogen. Die Beine liegen dann z.B. in Rückenlage übereinander oder überkreuzen sich sogar. Beim Gehen haben die Betroffenen dadurch erhebliche Schwierigkeiten. Bei chronischer Adduktoreninterferenz scheuern die Oberschenkel aneinander. Bei nicht gehfähigen Patienten ist das Sitzen und Liegen sowie die tägliche Pflege und Intimhygiene beeinträchtigt. Ist die Hüftmuskulatur betroffen, kann die chronische muskuläre Hyperaktivität zu einer progredienten Lateralisation des Hüftkopfs und unter Umständen sogar zur Hüftluxation und damit zu Immobilisation und Schmerzen führen. Bei Kindern mit Adduktorenspastizität ist die nor- male Hüftentwicklung durch die pathologische Belastung des wachsenden Gelenkes gefährdet.

Durch die Behandlung mit Botulinumtoxin können die motorischen Fähigkeiten der Patienten verbessert, Pflege und Intimhygiene der Patienten erleichtert werden (14, 15). Bei noch gehfähigen Patienten kann bereits nach einer Injektion eine breitere Schrittbasis erzielt und das Gehen erheblich erleichtert werden. Sind Hüft- und Kniebeugemuskulatur gleichzeitig von einer Adduktorenspastizität betroffen, kann jedoch in einigen Fällen die erforderliche Butulinumtoxin-Dosierung so hoch sein, dass eine intrathekale Baclofenapplikation oder eine Kombination von Botulinumtoxin mit einer oralen Pharmakotherapie sinnvoll ist.

Bei Adduktorenspastizität wird zur Auswahl der Zielmuskeln neben der Untersuchung und passivem Bewegen des Hüftgelenkes auch die Palpation der Adduktorenmuskulatur (Mm. Adductores longus, brevis und magnus) in unterschiedlichen Ausgangsstellungen empfohlen. Bei Hüftadduktion ist die mediale ischiokrurale Muskulatur (M. semimembranosus, M. semitendinosus) und gegebenenfalls der M. iliopsoas beteiligt. In der Regel sind bei Adduktorenspastizität bei zusätzlicher Hüftbeugung der M. adduktor brevis und longus und bei nur leichter Hüftbeugung auch der M. adductor magnus einbezogen. Aus prakti-

\section{Tab. 3 Adduktoren- und Beugespastik}

\begin{tabular}{|lllll}
\hline Muskel & $\begin{array}{l}\text { Anzahl der } \\
\text { Injektions- } \\
\text { stellen }\end{array}$ & $\begin{array}{l}\text { Dosis Botox } \\
\text { (Units) }\end{array}$ & $\begin{array}{l}\text { Dosis Dysport } \\
\text { (Units) }\end{array}$ & $\begin{array}{l}\text { Dosis Xeomin }^{\circledR} \\
\text { (Units) }\end{array}$ \\
\hline M. iliopsoas & $2-3$ & $50-100$ & $250-500$ & $50-100$ \\
\hline M. adductor longus & $1-2$ & $50-75$ & $250-375$ & $50-75$ \\
\hline M. adductor brevis & $1-2$ & $50-75$ & $250-375$ & $50-75$ \\
\hline M. adductor magnus & $1-3$ & $50-75$ & $250-375$ & $50-75$ \\
\hline M. gracilis & $1-2$ & $50-75$ & $250-375$ & $50-75$ \\
\hline M. biceps femoris & $2-3$ & $50-75$ & $250-375$ & $50-75$ \\
\hline M. semitendinosus & $1-2$ & $50-100$ & $250-600$ & $50-100$ \\
\hline M. semimembranosus & $1-2$ & $50-100$ & $250-500$ & $50-100$ \\
\hline Maximale Gesamtdosis & & 400 & 1000 & 300 \\
\hline Empfohlene Verdünnungen & $2-5 \mathrm{ml} /$ & $2-4 \mathrm{ml} /$ & $2-5 \mathrm{ml} /$ \\
& & 100 Units & 500 Units & 100 Units \\
\hline
\end{tabular}

* nicht zugelassen in dieser Indikation

Es sind nur die Dosierungen für eine Seite angegeben. In den allermeisten Fällen sind aber beidseitige Injektionen nötig modifiziert nach (21) 
schen Gründen sollten daher alle drei Muskeln beidseits an mindestens einem Injektionspunkt pro Muskel behandelt werden (Tab. 3).

Aufgrund der Anzahl und Größe der beteiligten Muskeln und des häufig ausgeprägten subkutanen Fettgewebes sollten die Injektionen Sonografie- bzw. EMG-gesteuert werden, besonders für ungeübte Behandler. In der Regel wird bei großen Muskeln an mehreren Stellen injiziert.

\section{Antikörperbildung}

Wie jeder körperfremde Wirkstoff kann auch die Injektion mit Botulinumtoxin die Bildung von Antikörpern hervorrufen. So genannte neutralisierende Antikörper können die Wirksamkeit der Injektion dann vermindern. In wieweit die Bildung von neutralisierenden Antikörpern bei Spastik vorkommt, kann bisher jedoch noch nicht eindeutig beantwortet werden. Man schätzt, dass bei Dystonie etwa 35\% der behandelten Patienten Antikörper bilden, allerdings liegt die Inzidenz neutralisierender Antikörper deutlich darunter. Es wird prospektiven Studien vorbehalten sein, die Frage der Inzidenz von neutralisierenden Antikörpern von neuen Toxinen wie Xeomin ${ }^{\circledR}$ bei Spastik zu klären.

Treatment of Spasticity with Botulinum Toxin Type $A$

Local intramuscular injections of botulinum toxin type A are currently being applied routinely in the treatment of focal dystonias and spastic syndromes. The resulting reduction in tonus appreciably reduces cumbering movement disorders and associated pain for a period of three to six months. Self-management by the patients of personal care and hygiene is thus facilitated, and their quality of life improved. Apart from the approved indications, such as hemifacial spasm, blepharospasm, hand or arm spasticity in adult stroke patients, the available data also support a recommendation for its use in other indications, too. The application of botulinum toxin in spasticity of the upper and lower extremities as also adductor spasticity is described.

\section{Key words}

botulinum toxin - cerebral palsy spasticity - stroke

\section{Literatur}

1. Arbeitskreis Botulinumtoxin der Deutschen Gesellschaft für Neurologie. Stellungnahme zur Kostenerstattung von Botulinumtoxin bei „Off-Label-Indikationen“ durch die gesetzlichen Krankenkassen vom 26.07.2004. www.botulinumtoxin.de

2. Bakheit AM, Thilmann AF, Ward AB, Poewe W, Wissel J, Muller J, Benecke R, Collin C, Muller F, Ward CD, Neumann C. A Randomized, Double-Blind, Placebo-Controlled, Dose-Ranging Study to Compare the Efficacy and Safety of Three Doses of Botulinum Toxin Type A (Dysport) With Placebo in Upper Limb Spasticity After Stroke. Stroke 2000; 31(10): 2402-6

3. Berweck S, Feldkamp A, Francke A, Nehles J, Schwerin A, Heinen F. Sonographyguided injection of botulinum toxin $\mathrm{A}$ in children with cerebral palsy. Neuropediatrics 2002; 33(4): 221-3

4. Bhakta BB, Cozens JA, Bamford JM, Chamberlain MA. Use of botulinum toxin in stroke patients with severe upper limb spasticity. J Neurol Neurosurg Psychiatry 1996; 61(1): 30-5

5. Boutsen F, Cannito MP, Taylor M, Bender B. Botox treatment in adductor spasmodic dysphonia: a meta-analysis. J Speech Lang Hear Res 2002; 45: 469-481

6. Brashear A, Gordon MF, Elovic E, Kassicieh VD, Marciniac C, Do M, Lee CH, Jenkins $\mathrm{S}$, Turkel C. Intramuscular injection of botulinum toxin for the treatment of wrist and finger spasticity after a stroke. N Engl J Med 2002; 347: 395-400

7. Cole R, Hallett M, Cohen LG. Doubleblind trial of botulinum toxin for treatment of focal hand dystonia. Mov Disord 1995; 10: 466-471

8. de Paiva A, Meunier FA, Molgo J, Aoki $\mathrm{KR}$, Dolly JO. Functional repair of motor endplates after botulinum neurotoxin type $A$ poisoning: biphasic switch of synaptic activity between nerve sprouts and their parent terminals. Proc Natl Acad Sci U S A 1999; 96(6): 3200-5

9. Deutsche Gesellschaft für Neurologie. Leitlinie „Dystonie“ 2002. www.dgn.org/ leitl.shtml

10. Gracies J-M, Hefter H, Simpson DM, Moore AP. Spasticity in adults. In: Moore AP, Naumann M (ed.). Handbook of Botulinum Toxin Treatment. Oxford: Blackwell Science, 2003: 219-271

11. Heinen F, Wissel J, Philipsen A, Mall V, Leititis JU, Schenkel A, Stucker R, Korinthenberg $R$. Interventional neuropediatrics: treatment of dystonic and spastic muscular hyperactivity with botulinum toxin A. Neuropediatrics 1997; 28(6): 307-13

12. Hesse $S$, Werner $C$. Poststroke motor dysfunction and spasticity: novel pharmacological and physical treatment strategies. CNS Drug 2003; 17(15): 1093-107

13. Lance JW. Spasticity: disordered motor control. In: Feldman RG, Young RR, Koella WP (ed.). Symposium synopsis. Chicago:
Yearbook Medical, 1980: 485-494

14. Mall V, Heinen F, Kirschner J, Linder M, Stein S, Michaelis U, Bernius P, Lane M, Korinthenberg R. Evaluation of botulinum toxin A therapy in children with adductor spasm by gross motor function measure. J Child Neurol 2000; 15(4): 214-7

15. Mall V, Heinen F, Siebel A, Korinthenberg R. Efficacy of BTX/A in the treatment of adductor spasticity: a randomized, double blinded, placebo-controlled trial. AACPDM 58th Annual Meeting, Los Angeles, 2004

16. Moore: Botulinum toxin A (Botulinumtoxin-A) for spasticity in adults. What is the evidence? Eur J Neurol 2002; 9 (Suppl 1): 42-47; 53-61

17. Naumann M, Jankovic J. Safety of Botulinum Toxin Type A: A Systematic Review and Meta-Analysis. Curr Med Res Opin 2004; 20(7): 981-990

18. Pittock SJ, Moore AP, Hardiman O, Ehler E, Kovac M, Bojakowski J, Al Khawaja I, Brozman M, Kanovsky P, Skorometz A, Slawek J, Reichel G, Stenner A, Timerbaeva S, Stelmasiak Z, Zifko UA, Bhakta B, Coxon E. A double-blind randomised placebo-controlled evaluation of three doses of botulinum toxin type $A$ (Dysport) in the treatment of spastic equinovarus deformity after stroke. Cerebrovasc Dis 2003; 15(4): 289-300

19. Tan EK, Jankovic J. Botulinum toxin A in patients with oromandibular dystonia: longterm follow-up. Neurology 1999; 53: 21022107

20. Ward AB, Aguilar M, De Beyl Z, Gedin S, Kanovsky P, Molteni F, Wissel J, Yakovleff A. Use of botulinum toxin type $A$ in management of adult spasticity - a European consensus statement. J Rehabil Med 2003; 35(2): 98-9

21. Wissel J, Naumann M. Fokale Behandlung der Spastizität mit Botulinumtoxin. In: Ochs G. (Hrsg.). Die Behandlung der schweren Spastizität. Stuttgart: Georg Thieme Verlag, 2004

Korrespondenzadresse:

Ao. Univ.-Prof. Dr. J. Wissel

Kliniken Beelitz GmbH

Neurologische Rehabilitationsklinik

Paracelsusring $6 a$

14547 Beelitz-Heilstetten

wissel@rehaklinik-beelitz.de 